\title{
In silico identification and characterization of the ion transport specificity for P-type ATPases in the Mycobacterium tuberculosis complex
}

\author{
Lorena Novoa-Aponte' ${ }^{1}$ Andrés León-Torres ${ }^{1}$, Miyer Patiño-Ruiz ${ }^{1}$, Jenifer Cuesta-Bernal ${ }^{1}$, Luz-Mary Salazar ${ }^{1}$, \\ David Landsman², Leonardo Mariño-Ramírez ${ }^{2,3 *}$ and Carlos-Yesid Soto ${ }^{1}$
}

\begin{abstract}
Background: P-type ATPases hydrolyze ATP and release energy that is used in the transport of ions against electrochemical gradients across plasma membranes, making these proteins essential for cell viability. Currently, the distribution and function of these ion transporters in mycobacteria are poorly understood.

Results: In this study, probabilistic profiles were constructed based on hidden Markov models to identify and classify P-type ATPases in the Mycobacterium tuberculosis complex (MTBC) according to the type of ion transported across the plasma membrane. Topology, hydrophobicity profiles and conserved motifs were analyzed to correlate amino acid sequences of P-type ATPases and ion transport specificity. Twelve candidate P-type ATPases annotated in the M. tuberculosis H37Rv proteome were identified in all members of the MTBC, and probabilistic profiles classified them into one of the following three groups: heavy metal cation transporters, alkaline and alkaline earth metal cation transporters, and the beta subunit of a prokaryotic potassium pump. Interestingly, counterparts of the non-catalytic beta subunits of Hydrogen/Potassium and Sodium/Potassium P-type ATPases were not found.

Conclusions: The high content of heavy metal transporters found in the MTBC suggests that they could play an important role in the ability of $M$. tuberculosis to survive inside macrophages, where tubercle bacilli face high levels of toxic metals. Finally, the results obtained in this work provide a starting point for experimental studies that may elucidate the ion specificity of the MTBC P-type ATPases and their role in mycobacterial infections.
\end{abstract}

Keywords: Tuberculosis, Mycobacterium tuberculosis complex, P-type ATPases, Ion transport, Conserved motifs

\section{Background}

Tuberculosis (TB) is one of the most important challenges in public health maintenance throughout the world. According to the World Health Organization (WHO), 8.5-9.2 million new TB cases were estimated to have occurred in 2010 [1], and 1.2-1.5 million deaths were caused by species of the Mycobacterium tuberculosis complex (MTBC) that includes M. tuberculosis, M. bovis, $M$. bovis BCG (vaccine strain), $M$. africanum, M. microti, M. canettii, and M. pinnipedii, which produces TB in humans and some animal hosts [2,3]. Part of the infected population will develop active TB,

\footnotetext{
* Correspondence: marino@ncbi.nlm.nih.gov

${ }^{2}$ Computational Biology Branch, NCBI, NLM, NIH, Bethesda, USA

${ }^{3}$ PanAmerican Bioinformatics Institute, Santa Marta, Magdalena, Colombia

Full list of author information is available at the end of the article
}

whereas the majority of cases (approximately 90\%) progress to a non-infectious disease or latent $\mathrm{TB}$, where mycobacteria survive in a dormant state inside immune cells [4]. Individuals with latent TB may be asymptomatic during prolonged periods of time; however, $\mathrm{TB}$ can reactivate when the host immune response diminishes due to malnutrition, steroid use, and HIV co-infection [5].

The emergence of multidrug and extensively drugresistant tuberculosis strains (MDR-TB and XDR-TB) and the lack of drugs against latent TB have become serious problems for TB control. Therefore, the identification of new therapeutic targets useful in the development of novel drugs and vaccines against latent TB is essential. New anti-TB drugs, such as diarylquinolines (TMC207) and benzothiazines (BTZ043) target

\section{Biomed Central}


essential membrane proteins that affect mycobacterial viability [6]. Thus, antimicrobials designed against proteins of plasma membrane are ideal because they avoid problems related to membrane permeability.

Ion transport in bacteria is carried out by enzymatic systems that belong to either the P-type ATPase, ATP binding cassettes (ABC transporters) and metallic ion $/ \mathrm{H}^{+}$-antiporter systems [7]. In general, ATPases help maintain the ion gradients responsible for cell volume control and transport of nutrients across the cell membrane [8-11]. ATPases hydrolyze ATP, releasing energy that is used in the transport of ions against electrochemical gradients in plasma membranes. The enzymatic mechanisms of P-type ATPases were initially described in eukaryotic cells [12-14]. These enzymes have five different functional and structural domains: three of these domains are cytoplasmic (A, actuator; $\mathrm{N}$, nucleotide binding and $\mathrm{P}$, phosphorylation), and the other two are embedded in the membrane ( $T$, transport and $S$, class specific support domain) [15]. P-type ATPases have the following two conformational states: E1, which binds ion substrates on one side of the cell membrane, inducing their autophosphorylation and generating a new conformational state; and E2, which has a lower affinity for substrates and therefore releases them to the other side of the cell membrane, promoting ion transport and finally recovery of the E1 state $[15,16]$.

Despite P-type ATPases share the same catalytic mechanism based on conformational changes in their five structural domains, their regulation and substrate affinities are different $[15,16]$. P-type ATPases are phylogenetically classified into five subfamilies $\left(\mathrm{P}_{\mathrm{I}}-\mathrm{P}_{\mathrm{V}}\right)$, and within these subfamilies are 10 different subtypes that are categorized based on the transported substrate [17].

In this study, probabilistic profiles were constructed to compare and classify all P-type ATPases of the MTBC based on their structural features and ion transport. Twelve possible P-type ATPases were detected in the proteome of $M$. tuberculosis $\mathrm{H} 37 \mathrm{Rv}$ and from other members of the MTBC. The high number of heavy metal transporters discovered in the MTBC suggests an important role for P-type ATPases in M. tuberculosis survival within macrophages.

\section{Methods}

\section{Construction of hidden Markov models (HMM)}

To obtain a representative group of each phylogenetic subfamily, a set of 128 well-characterized P-type ATPases with evidence of existence at the protein level were retrieved from Uniprot (Swiss-Prot section) [18]. Each group of sequences was aligned using the Praline tool (http://www.ibi.vu.nl/programs/pralinewww/) with the BLOSUM62 matrix and Phobius transmembrane structure predictor, which was developed to improve the multiple alignments of membrane protein sequences [19]. The $H M M$ package of programs [20], available in the Mobyle Pasteur portal (http://mobyle.pasteur.fr/cgibin/portal.py\#welcome), was used to find these types of pumps in the MTBC proteomes. The Hmmbuild tool with default settings and the multiple sequence alignments was used for HMM building. The default parameters of the Hmmer tools use an ad hoc position-based sequence weighting algorithm that makes the models appropriate for the identification of distant members of the P-type ATPases family. Consensus sequences were generated with the Hmmemit tool.

\section{Search and classification of MTBC P-type ATPases}

To date, the following 10 MTBC genomes have been completely sequenced and assembled (NCBI): $M$. africanum GM041182 (NC_015758), M. bovis AF2122/97 (NC_002945), M. bovis BCG str. Pasteur 1173P2 (NC_008769), M. bovis BCG str. Tokyo 172 (NC_012207), M. canettii CIPT 140010059 (NC_015848) and five M. tuberculosis strains, H37Rv (NC_000962), H37Ra (NC_009525), F11 (NC_009565), CDC1551 (NC_002755) and KZN1435 (NC_012943); these proteomes were obtained from Uniprot (http://www.uniprot. org/). Because strains of M. microti and M. pinnipedii had not been sequenced, they were not included in this study. HMM and Hmmsearch tool were used to find P-type ATPases in the MTBC proteomes.

\section{Topology prediction}

The topology derived from predictions of transmembrane segments (TMS) was made with the following six programs: TopPred (http://mobyle.pasteur.fr/cgi-bin/portal. py\#forms::toppred), DAS (www.sbc.su.se/ miklos/DAS/), TMpred (www.ch.embnet.org/software/TMPRED_form. html), TMHMM 2.0 (www.cbs.dtu.dk/services/TMHMM), HMMTOP (www.enzim.hu/hmmtop/) and Phobius (phobius.sbc.su.se/). All programs were used with default settings, except TopPred, which allows the user to provide information about the type of organism. TMDET and $P P M$ servers were used in cases where the predictions based on amino acid sequences did not yield reliable results. These tools must be fed with PDB files to identify TMS based on tertiary protein structure and to generate 3D models with TMS located into hypothetical lipid bilayer planes. Tertiary structure models of $\mathrm{CtpH}$ and CtpI were made with the I-TASSER tool [21] based on the threading strategy and were validated with the Whatif package of programs (http://swift.cmbi.ru.nl/servers/html/ index.html).

\section{Hydrophobicity profile construction}

The amino acid sequence of the M. tuberculosis H37Rv P-type ATPases and consensus sequences generated by Hmmemit tool were analyzed by TMHMM 2.0. 
Hydrophobicity profiles of consensus sequences for previously characterized P-type ATPases were used as comparison patterns.

\section{Conserved motif analysis}

Amino acids sequences of the identified proteins were manually analyzed to determine the nine conserved motifs typical of the P-type ATPase family, as previously described by Thever et al. [22] and others [16,23].

\section{Results and discussion}

An unusually high number of cation transporter P-type ATPases are present in the Mycobacterium tuberculosis complex

Multiple alignments of 128 reported P-type ATPase protein sequences from a representative group of eukaryotic and prokaryotic cells (obtained from a curated database and confirmed at the protein level) allowed the identification of highly conserved regions within the family and the classification of these members according to ion transport. These alignments were used as the starting point for the construction of HMM profiles that represented groups of ion transporters, which were also used to generate a consensus sequence for each group. The designed HMM were then used to identify P-type ATPases in proteomes of the different MTBC species, whose genome sequences have been reported. The proposed classification for the studied sequences was based on the obtained scores using the HMM and the Hmmsearch tool.

Because the P-type ATPases are transmembrane proteins, typical alignments using BLOSUM and PAM matrices were not adequate. In this study, the Praline tool was used because it considers the differences in evolutionary tendencies of transmembranal and nontransmembranal regions. Praline applies an adequate matrix for each protein region based on the previous prediction of TMS (made with the Phobius algorithm in this case). As P-type ATPases contain ion-binding motifs within a transmembrane domain, the strategy that we employed produced more reliable alignments. Twelve hypothetical proteins with a high probability of being P-type ATPase transporters of metallic cations were identified in each of the MTBC proteomes. All proteins identified in the MTBC share at least 98\% identity with their orthologs in the scanned proteomes; however, $M$. canettii and $M$. africanum displayed slight differences in non-conserved regions. The pumps identified in the M. tuberculosis $\mathrm{H} 37 \mathrm{Rv}$ proteome, CtpA, CtpB, CtpC, CtpD, CtpE, CtpF, CtpG, CtpH, CtpI, CtpJ, CtpV and $\mathrm{KdpB}$, were in agreement with the automatic annotation of probable cation transporter P-type ATPases in the M. tuberculosis H37Rv genome [24] and were taken as references to facilitate further analysis of results.

\section{A broad diversity of cations are potentially transported by Mycobacterium tuberculosis P-type ATPases}

Figure 1 shows the Hmmsearch scores obtained when the 16 HMM were used to find P-type ATPases in the M. tuberculosis $\mathrm{H} 37 \mathrm{Rv}$ proteome. Those scores show the similarity between the identified sequences in the H37Rv proteome and the grouped sequences used for each HMM construction. The patterns thus obtained allowed classification of the 12 P-type ATPases into the following three groups: transporters of heavy metal (HM) cations, transporters of alkaline and alkaline earth metal (AEM) cations, and a group composed only of the $\beta$ subunit of the prokaryotic $\mathrm{K}^{+}$transporter, $\mathrm{KdpB}$. The highest similarity with the conventional P-type ATPases used in this study corresponded to $\mathrm{KdpB}, \mathrm{CtpF}$ and $\mathrm{CtpV}$, whereas CtpE, CtpH and CtpI had the lowest scores.

The HM group is composed of CtpA, CtpB, CtpC, CtpD, CtpG, CtpJ and CtpV, which may transport $\mathrm{Cu}^{2+}$, $\mathrm{Cu}^{+}, \mathrm{Co}^{2+}, \mathrm{Ag}^{+}, \mathrm{Hg}^{2+}, \mathrm{Cd}^{2+}, \mathrm{Pb}^{2+}$ and $\mathrm{Zn}^{2+}$. Most of the proteins analyzed in this work correspond to the HM group (60\%) suggesting that the active transport of heavy metal cations is relevant for the tubercle bacilli persistence, as it has been hypothesized for other prokaryotes and some unicellular eukaryotes [23]. Interestingly, evidence of toxic concentrations of intracellular heavy metals has been recently described in macrophages infected with $M$. tuberculosis [25].

Alternatively, CtpE, CtpF, CtpH and CtpI belong to the AEM group and may be involved in $\mathrm{Na}^{+}, \mathrm{K}^{+}, \mathrm{Ca}^{2+}$, $\mathrm{H}^{+}$and $\mathrm{Mg}^{2+}$ transport. It is possible that some of these proteins correspond to $\mathrm{Na}^{+} / \mathrm{K}^{+}$or $\mathrm{H}^{+} / \mathrm{K}^{+}$ATPase pumps, but they only appeared to contain the $\alpha$ subunits in their tridimensional structure. Their non-catalytic- $\beta$ subunit counterpart, which has been correlated with regulatory processes and assembly of P-type ATPases into the cell membrane of eukaryotic cells [10], was not found in any of the MTBC proteomes.

$\mathrm{KdpB}$ is very different protein from the other MTBC ATPases. It shares $63 \%$ identity with the $\mathrm{KdpB}$ of $E$. coli (P03960), which corresponds to the $\beta$ subunit of a $\mathrm{K}^{+}$ transporting multimeric ATPase [22,26]. Despite the observation that $\mathrm{KdpB}$ has the characteristic DKTGTLT phosphorylation motif of this type of pump, it does not have a typical ion binding motif [26]. In conclusion, the proposed classification for P-type ATPases of the MTBC provides an initial approximation of their functional characterization. It is noteworthy that the constructed HMM in this work became a useful tool for the identification of P-type ATPases in other biological systems.

\section{Three different topologies can be adopted by P-type ATPases in Mycobacterium tuberculosis}

The six different algorithms used in the hydrophobicity analysis showed that all of the M. tuberculosis H37Rv 


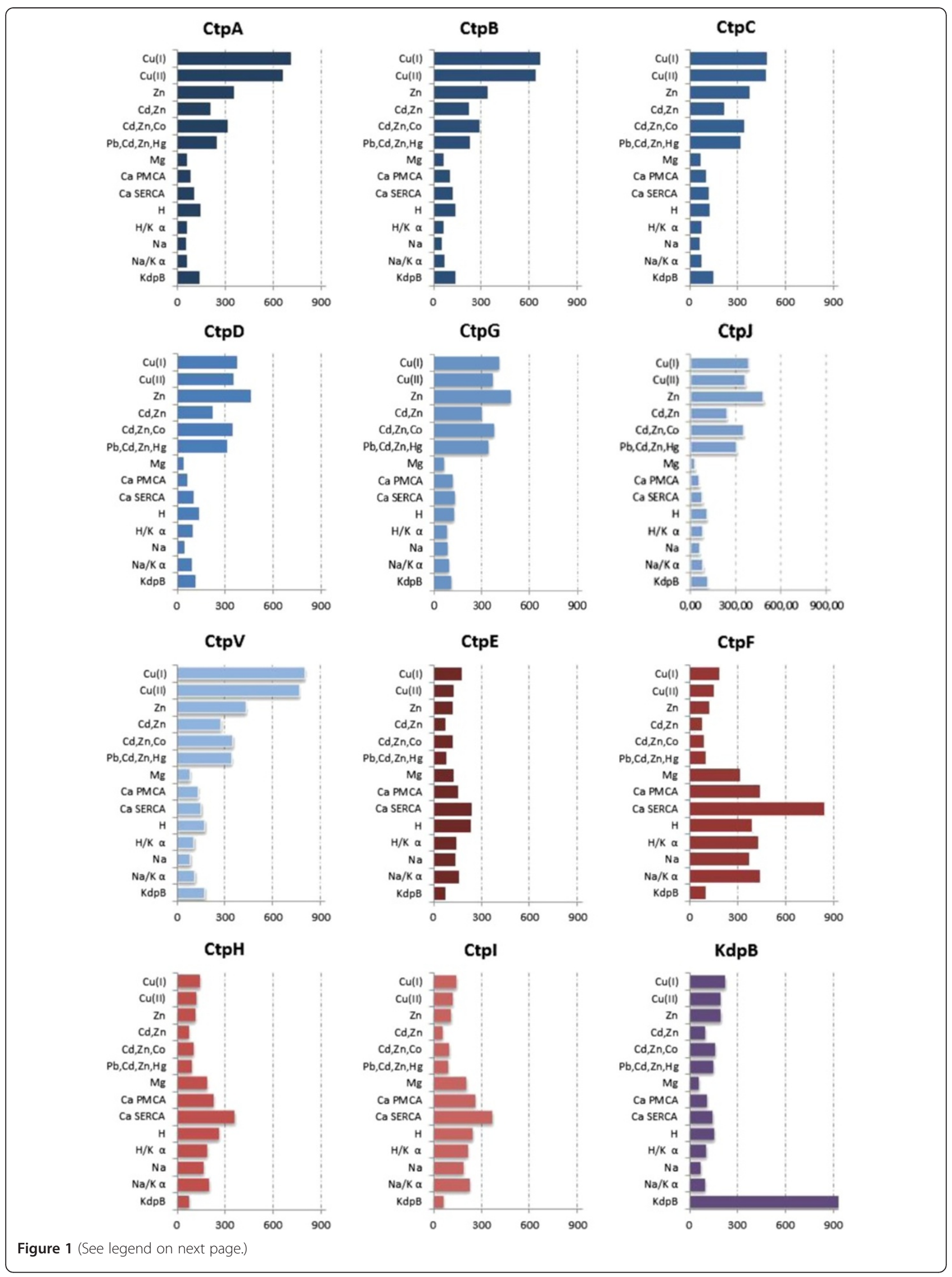


P-type ATPases have an $\alpha$-helix type TMS containing at least 17 amino acid residues, and the agreement of the topology results obtained using these prediction tools increases the confidence in the predicted TMS. Consensus transmembrane regions for each P-type ATPase were obtained if at least four of the algorithms gave similar results. Because three of the tools (TMHMM 2.0, HMMTOP and Phobius) incorporate size and composition restrictions in TMS, the results obtained using these algorithms are significant.

The strategy used for topology analysis showed the following three different types of topology within the M. tuberculosis H37Rv P-type ATPases: type I, which corresponds to HM pumps with eight TMS (A, B and from 1 to 6), type II, which corresponds to AEM pumps with 10 TMS (from 1 to 10), and type III, which corresponds to KdpB with 7 TMS (Figure 2). It was observed that all P-type ATPases have two cytoplasmic loops (small and large) that include the phosphorylation and ATP binding sites. The small cytoplasmic loop is located between TMS2 and TMS3, whereas the largest cytoplasmic loop is situated between TMS4 and TMS5 (Figure 2) [22]. Additionally, as was expected, the $\mathrm{N}$ - and C-termini of these proteins are located in the cytosolic side, except in the case of $\mathrm{KdpB}$, in which the $\mathrm{N}$ - terminus is located intracellularly and the $\mathrm{C}$ - terminus is outside the cytoplasm.

Disagreement in the results was observed in $\mathrm{CtpH}$ and CtpI analysis using the different prediction tools. CtpI showed some hydrophobic amino acid sequences that could be considered part of TMS, but they do not fulfill all of the characteristics associated with TMS; meanwhile, $\mathrm{CtpH}$ did not show the expected hydrophobicity pattern for P-type ATPases (Figure 3). Therefore, at first it was difficult to determine whether $\mathrm{CtpH}$ and CtpI were in fact typical of P-type ATPases. Recent studies have classified CtpH and CtpI proteins of M. bovis as FUPA 24 (TC No. 3.A.3.24), i.e., "Functionally uncharacterized P-type ATPase family 24 ", and classify them as transporter proteins in the TCDB-Transporter Classification Database (http://www. tcdb.org). FUPA 24 proteins are homologous to P-type ATPases but have an unusually large $\mathrm{N}$-terminal segment that makes them twofold longer than typical P-type ATPases [23] with two TMS. In addition, functional motifs of P-type ATPases are located within the C-terminal region of FUPA 24 [23].
By contrast, topology analysis in this study showed that $M$. tuberculosis $\mathrm{H} 37 \mathrm{Rv} \mathrm{CtpH}$ and CtpI contain more than the expected TMS (from 3 to 12) for FUPA 24 proteins. To overcome the discrepancy between TCDB and the six topology tools used in this work, the TMDET and PPM servers were used. These tools allow for the identification of TMS based on the tertiary structure of the protein; thus, not only can the correct size and composition of $\alpha$-helixes be guaranteed, but also their adequate disposition and organization in the plasma membrane can be determined. Ten TMS were found in $\mathrm{CtpH}$ and CtpI using both the TMDET and PPM servers. These segments are located with high probability in a hypothetical lipid bilayer as shown in Figure 4. This result strongly suggests that $\mathrm{CtpH}$ and CtpI have a type II topology, as has been determined for other members of the AEM group.

\section{Hydrophobicity profiles suggest insights into ion transport specificity}

The "topological clustering" based on the hydrophobicity profiles obtained with the TMHMM 2.0 algorithm can be used as a predictive tool to determine substrate specificity for HM pumps [27]. Here, this strategy was applied to analyze HM, AEM and KdpB groups of $M$. tuberculosis H37Rv P-type ATPases. The hydrophobicity profiles from each $M$. tuberculosis P-type ATPase was compared with the obtained profiles from previously characterized P-type ATPases. As shown in Figure 3, the hydrophobicity profiles of CtpA, CtpB and CtpV were similar to the consensus $\mathrm{Cu}^{+} \mathrm{P}$-type ATPases; the $\mathrm{CtpC}$ profile was similar to the different $\mathrm{Zn}^{2+}$ transporters $\mathrm{Zn}^{2+}, \mathrm{Cd}^{2+} / \mathrm{Zn}^{2+}$ and $\mathrm{Pb}^{2+} / \mathrm{Cd}^{2+} / \mathrm{Zn}^{2+} / \mathrm{Hg}^{2+}$. In addition, CtpD, CtpG and CtpJ had regions similar to the $\mathrm{Cd}^{2+} / \mathrm{Zn}^{2+} / \mathrm{Co}^{2+}$ transporter. In the case of the AEM group, CtpF possesses TMS that were similar to the hydrophobic profiles obtained for consensus of $\mathrm{Na}^{+}$, $\mathrm{Mg}^{2+}$ and $\mathrm{Na}^{+} / \mathrm{K}^{+}$ATPases. Additionally, CtpE was similar to $\mathrm{Na}^{+}$or $\mathrm{H}^{+}$transporters; however, its hydrophobicity profile is closer to that of $\mathrm{Na}^{+}$P-type ATPase. Considering the C-terminus region of $\mathrm{CtpH}$ (819 amino acid residues), this pump had a hydrophobicity profile very similar to the $\mathrm{Ca}^{2+}$ PMCA consensus. Finally, KdpB had the exact same hydrophobic profile as that which was determined for E. coli KdpB. 


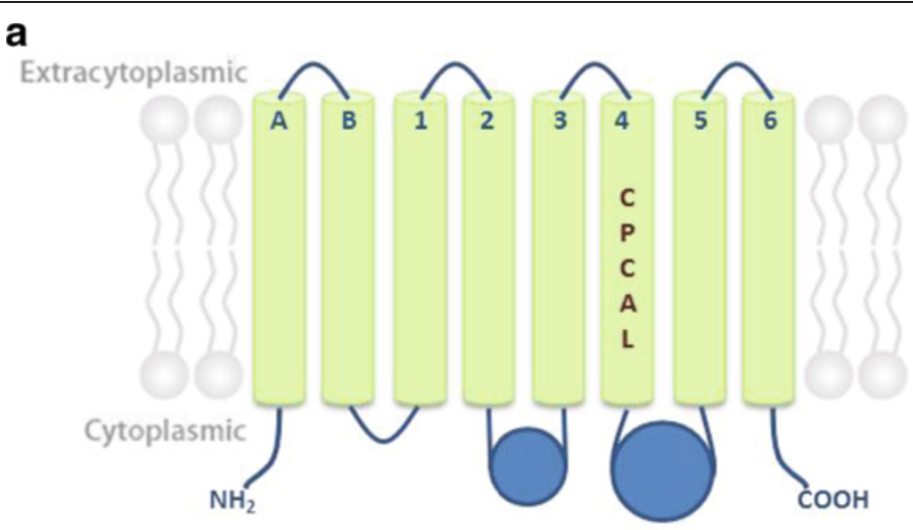

b
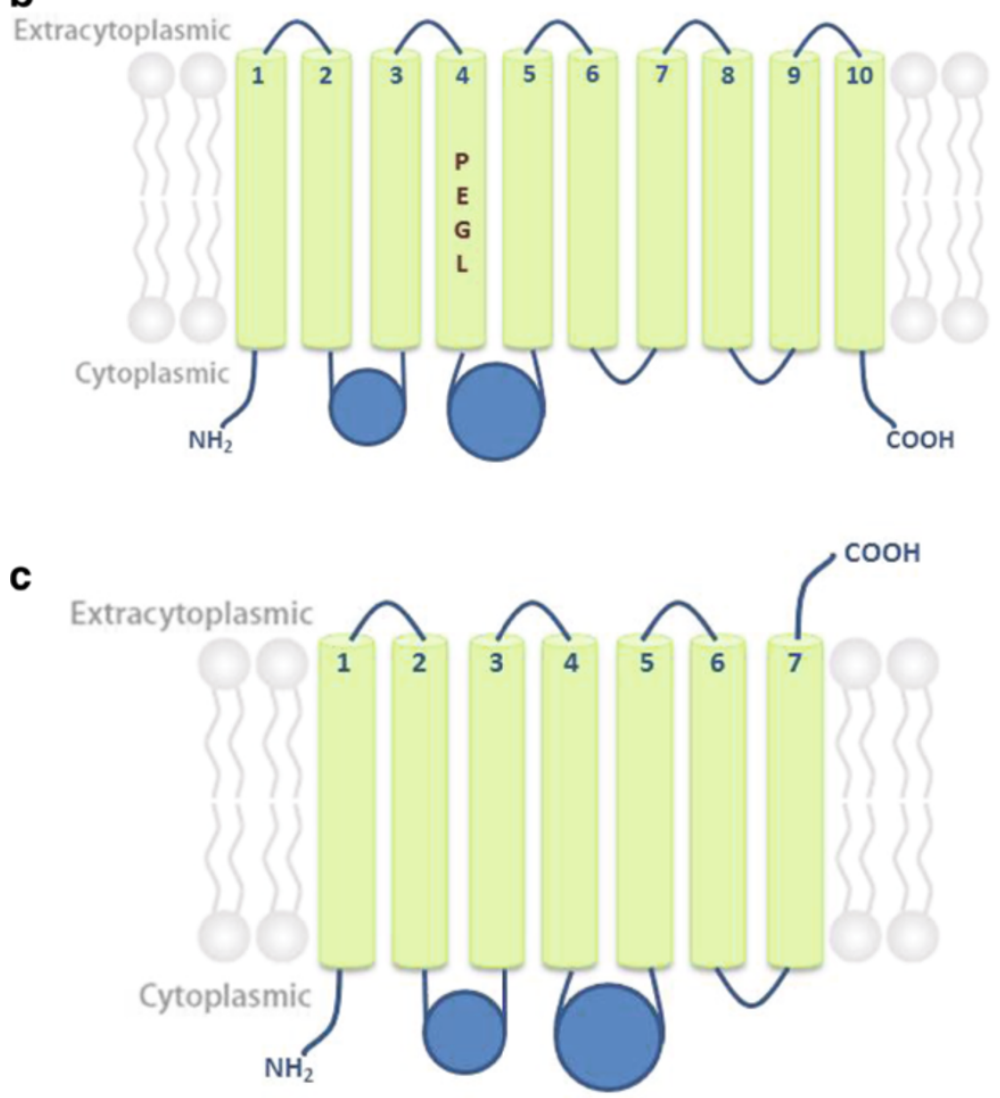

Figure 2 Predicted topology for P-type ATPases of MTBC. Type I topology (HM group ATPases) (a), type II topology (EAM group ATPases) (b), type III topology (KdpB group ATPases) (c), lon binding motifs (number 4) are indicated in the corresponding transmembrane domain.

In general, there are the following two types of hydrophobicity profiles: the first comprises the AEM and the second that corresponds to HM. Interestingly, the relative position of TMS along amino acid sequences is highly conserved between both types of hydrophobicity profiles. The profile for AEM is characterized by a single TMS in the C-terminal region and two in the $\mathrm{N}$-terminal region, whereas profiles for HM P-type ATPases have three highly hydrophobic regions, one of them in the $\mathrm{N}$-terminus, one in the middle of the amino acid sequence, and the third in the C-terminal end. Although the hydrophobicity profile of consensus sequences is similar among enzymes in each group, there are significant differences between profiles that allow their differentiation according to the transported ion.

\section{P-type ATPases of the Mycobacterium tuberculosis complex possess the characteristic motifs involved in cation transport}

The common catalytic mechanism of P-type ATPases is partially supported by conserved core sequences that are 


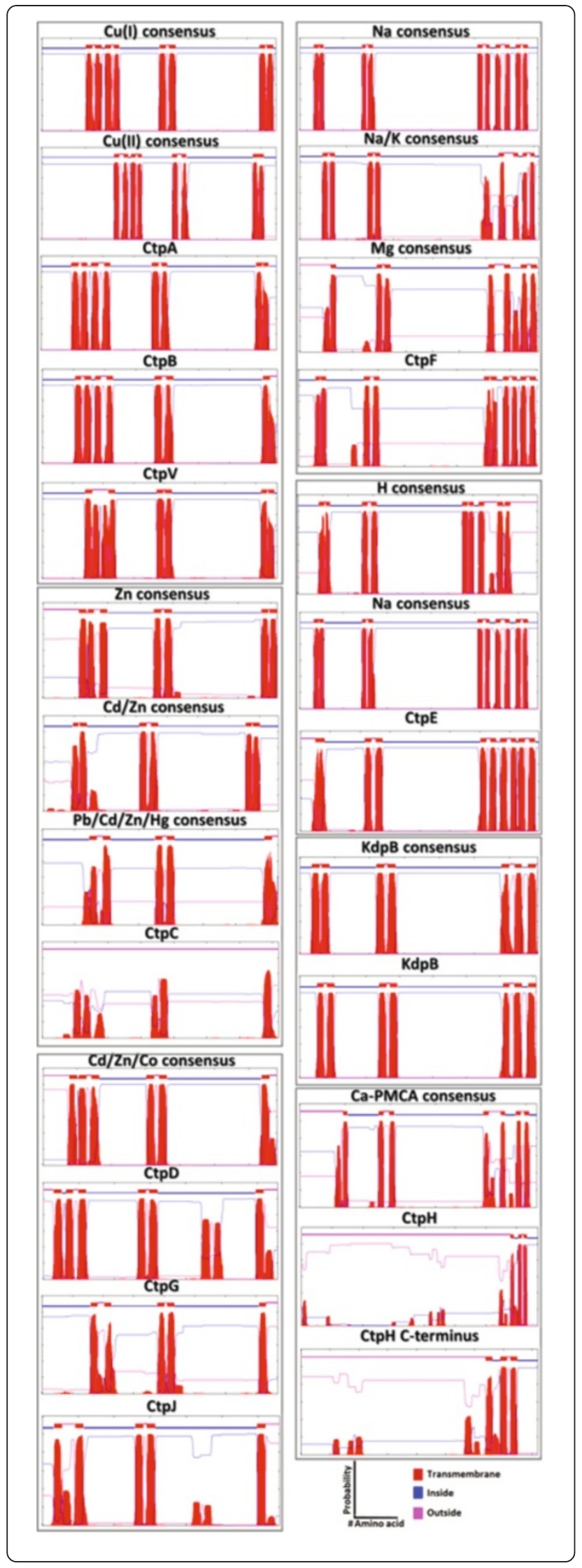

Figure 3 Hydrophobicity profiles of $M$. tuberculosis H37Rv P-type ATPases. Hydrophobicity profiles allow grouping of P-type ATPases with seven different ion specificities.

useful in the recognition of these pumps in proteomes [15]. To find the conserved motifs for P-type ATPases along the protein sequences, manual analysis was carried out because the servers commonly used for analysis of conserved motifs do not identify subtle variations within particular motifs of P-type ATPases. Nine conserved motifs typical for P-type ATPases were found in the MTBC pumps (Figure 5). We observed that each motif sequence was identical within ortho$\operatorname{logs}$ of the MTBC. From $\mathrm{N}$-terminus to the $\mathrm{C}$ terminus, the motifs were as follows: motifs 1 [(PVA)G(DE)] and 2 [P(AS)D], related to conformational changes, together with motif 3 [TGE(SA)], associated with phosphatase activity, were located between TMS 2 and 3 in the actuator domain; the sequence of motif 4 , which determines ion specificity, can be $[\mathrm{PEG}(\mathrm{LM})]$ or $[(\mathrm{CSA}) \mathrm{PCA}(\mathrm{LV})]$, which was located at the end of the fourth TMS; the phosphorylation site [DKTGTLT] was found in the P domain; the remaining motifs, motif $6[(\mathrm{KI}) \mathrm{GA}(\mathrm{PVA})$ (EDA)], an ATP binding facilitator, motifs 7 [(DV) (ASPI)(VP)(KAR)] and 8 [(MLV)I(TS)GD], involved in phosphorylation catalysts, and motif 9 [(VTC)AM (TV)GDG(VSAT)ND(AV)(PAL)A(LI)(RKA)(QMAD)A $(\mathrm{DNT})(\mathrm{VI}) \mathrm{G}(\mathrm{VI})(\mathrm{AG})(\mathrm{MV})]$, the hinge motif, which provides the flexibility necessary to achieve conformational changes during the pumping process, could be found between the fourth and fifth TMS [22]. Motifs 8 and 9 contain amino acid residues [TGDN and GDGXND] responsible for the coordination of $\mathrm{Mg}^{2+}$ as a cofactor of the enzymes $[15,16,22,23]$.

It was observed that characteristic motifs of P-type ATPases exhibit slight variations compared with previously reported sequences for eukaryotic organisms [22]. The most conserved motif (motif 5) was almost the same within the 12 P-type ATPases, except CtpH and $\mathrm{KdpB}$, which contained the conservative substitutions [DKTGTL(TS)] and [DKTGT(LI)T], respectively. The sequence of motif 4 [PEGL] has been previously associated with binding of AEM cations [22], and it has been found in CtpE, CtpF, and CtpI. Variation in the fourth position of this motif [PEGM] in $\mathrm{CtpH}$ has not been reported previously. CtpA, CtpB, CtpC, CtpD, CtpJ, CtpG and CtpV have the motif 4 sequence [(CSA)PCA(LV)] characteristic of HM transporters. Alternatively, $\mathrm{KdpB}$, which corresponds to a $\beta$ subunit of a multimeric P-type ATPase, did not have motif 4 . This finding can be explained by the observation that this subunit mediates phosphorylation/dephosphorylation and 


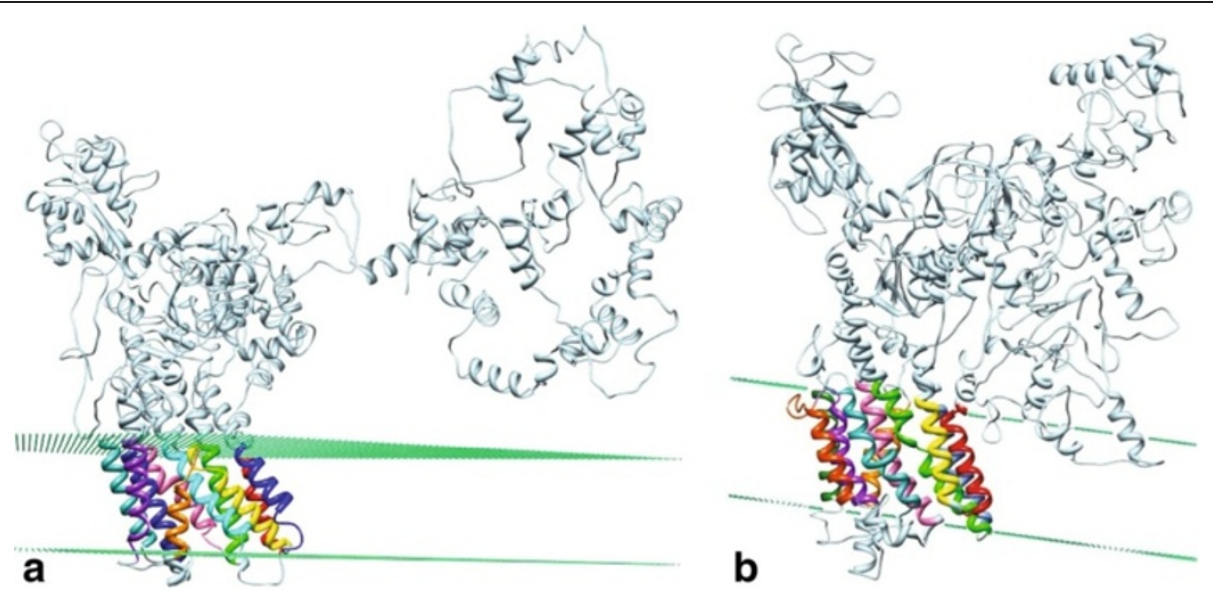

Figure 4 The CtpH and Ctpl mycobacterial P-type ATPases (type II topology) exhibit ten TMS in their C-terminal half (different color helixes). These tertiary structure models were generated with the I-TASSER tool and were modified with the PPM server [89] to include the possible location of the lipid bilayer. Dummies (green color) correspond to the location of the carbonyl groups in the bilayer. (a) CtpH model generated with the PPM server, (b) Ctpl model built based on the TMDET results.

energy transduction during $\mathrm{K}^{+}$transport. In E. coli, the coordination function lies mainly in the KdpA subunit [26].

\section{$\mathrm{Cu}^{+}, \mathrm{Zn}^{2+}$ and $\mathrm{Co}^{2+}$ pumping is mediated by P-type ATPases in the $M$. tuberculosis complex}

The $\mathrm{P}_{\mathrm{IB}}$ phylogenetic group of P-type ATPases is composed of HM pumps, and according to ion specificity, the $\mathrm{P}_{\mathrm{IB}}$ group is subdivided into five subgroups ( 1 to 5 ). Alignments of consensus sequences from characterized $\mathrm{P}_{\mathrm{IB}}$ ATPases with the HM P-type ATPases of MTBC were used for locating additional transmembrane motifs of this type of transporter [28-30], as shown in Figure 6. CtpA, CtpB and CtpV have the characteristic motifs of $\mathrm{P}_{\mathrm{IB}-1}$ group members (Table 1), corroborating their ion transport specificity as predicted by hydrophobicity profiles, and suggesting that $\mathrm{Cu}^{+}$is transported preferentially to $\mathrm{Cu}^{2+}$ by these pumps.

This result is expected, because the special reducing conditions inside mycobacteria [27] are similar to the intra-phagosomal environment in macrophages where the bacilli reside. Recently, it was reported that the M. tuberculosis $\mathrm{H} 37 \mathrm{Rv} \mathrm{CtpV}$ is a $\mathrm{Cu}^{+}$exporter P-type ATPase, reinforcing our predictions [31]. CtpD and CtpJ exhibit the $[\mathrm{SCP}]$ and [HEGT] motifs of the $\mathrm{P}_{\mathrm{IB}-4}$ group in the TMS6 and TMS8, respectively. Some reports have indicated that amino acids involved in $\mathrm{Co}^{2+}$ transport are still not well understood; for this reason, the absence of residue $\mathrm{N}$ in TMS7 cannot rule out the possibility that $\mathrm{CtpD}$ and $\mathrm{CtpJ}$ are $\mathrm{Co}^{2+}$ transporters. CtpC only exhibits the $\left[\mathrm{CPC}(\mathrm{X})_{4} \mathrm{~S}\right]$ motif in TMS6, which allows its classification as $\mathrm{P}_{\mathrm{IB}-2}$. In fact, it was recently reported as a $\mathrm{Zn}^{2+}$ P-type ATPase in M. tuberculosis H37Rv [25]; deficiency in this pump produced zinc accumulation within the mycobacterial cytoplasm, resulting in impaired intracellular growth of tubercle bacilli [25]. Finally, CtpG does not have any of the additional motifs present in TMS6, TMS7 and TMS8; however, it possesses the [WI(YE)(RG)] sequence just before TMS6, between positions 406 and 409, and the [LS] motif located on TMS7, which is associated with $\mathrm{Zn}^{2+}$ P-type ATPases [32,33].

All of the results from this work provide predictive evidence for experimental studies to establish the ion specificity of MTBC P-type ATPases and their role in mycobacterial infection. It has been observed that some P-type ATPases of tubercle bacilli are over-expressed under conditions that mycobacteria face during infection $[25,34,35]$. For example, the expression level of CtpF and CtpC increase when M. tuberculosis is exposed to isoxyl, tetrahydrolipstatine and SRI\#9190 antimicrobial compounds, indicating that these pumps might contribute to intrinsic resistance of mycobacteria to antimicrobial drugs [36]. Two additional studies indicate that hypoxia induces upregulation of the AEM transporter CtpF $[37,38]$; this observation is in agreement with those of another study that reported that CtpF is part of the regulon of the DosRS system, a relevant regulator of latency in M. tuberculosis [39]. In addition, upregulation of CtpF is observed in vitro when M. tuberculosis is incubated in the presence of S-nitrogluthatione GSNO, ethanol, $\mathrm{H}_{2} \mathrm{O}_{2}$ and nitric oxide [40]. Moreover, CtpA, CtpC, CtpG, CtpV and CtpF are also induced when $M$. tuberculosis is phagocytized by macrophages [25,34,35].

\section{Conclusion}

Mycobacteria are unicellular organisms that respond to environmental stimuli, and the transport of substances across the plasma membrane could play a fundamental role in their adaptability. Computational analysis shows 


\begin{tabular}{|c|c|c|c|c|c|c|c|c|c|}
\hline & 1 & 2 & 3 & 4 & 5 & 6 & 7 & 8 & 9 \\
\hline & PGD & PAD & TGES & PEGL & DKTGTLT & KGAPE & DPPR & MVTGD & VAVTGDGVNDSPALKKADIGVAM \\
\hline CtpA & PDG & PAD & TGEA & CPCAL & DKTGTLT & AATVE & DAVK & LLTGD & VAMVGDGINDGPALARADLGMAI \\
\hline Position & 251 & 259 & 296 & 399 & 443 & 473 & 566 & 588 & 632 \\
\hline CtpB & PGE & AAD & TGEA & CPCAL & DKTGTLT & AAAVE & DTLK & LLTGD & VAMVGDGINDGPALVGADLGLAI \\
\hline Position & 276 & 281 & 299 & 402 & 446 & 480 & 575 & 597 & 641 \\
\hline CtpC & IGD & PVD & TGEN & CPCAV & DKTGTLT & KKASE & DEVR & MLTGD & VGMVGDGINDAPALAAADIGIAM \\
\hline Position & 238 & 251 & 269 & 364 & 408 & 505 & 538 & 561 & 605 \\
\hline CtpD & VGD & PAD & TGES & SPCAV & DKTGTLT & RGAPL & DQLR & LLTGD & VLLVGDGVNDAPAMAAARAAVAM \\
\hline Position & 169 & 182 & 200 & 303 & 347 & 437 & 471 & 494 & 538 \\
\hline CtpE & PGD & VVD & TGEA & PEGL & DKTGTLT & IGAPD & DARE & VISGD & VAMTGDGVNDVLALKDADIGVAM \\
\hline Position & 129 & 134 & 153 & 258 & 301 & 386 & 447 & 464 & 531 \\
\hline CtpF & PGD & PAD & TGES & PEGL & DKTGTLT & KGAVE & DPPR & MITGD & VAMTGDGVNDAPALRQANIGVAM \\
\hline Position & 142 & 155 & 174 & 290 & 333 & 465 & 541 & 563 & 638 \\
\hline CtpG & VGD & ATD & TGES & APCAL & DKTGTLT & AAALE & DELR & MLTGD & TAMVGDGVNDAPALAAADLGIAM \\
\hline Position & 285 & 298 & 316 & 418 & 462 & 492 & 581 & 603 & 646 \\
\hline CtpH & PGD & PAD & TGES & PEGM & DKTGTLS & KGAPE & DTPR & LITGD & CAMVGDGSNDAAAIRAATVGIGV \\
\hline Position & 776 & 789 & 808 & 922 & 965 & 1060 & 1132 & 1154 & 1225 \\
\hline CtpI & VGD & PAD & TGES & PEGL & DKTGTLT & KGAPE & DTAR & LITGD & TAMVGDGANDAAAIRMADVGIGV \\
\hline Position & 865 & 878 & 897 & 1010 & 1053 & 1167 & 1243 & 1265 & 1335 \\
\hline CtpJ & IGD & SAD & TGEP & SPCAV & DKTGTLT & RGTPE & DQLR & LLTG D & LTVVGDGINDAPALAAAHVGIAM \\
\hline Position & 162 & 175 & 193 & 296 & 340 & 347 & 472 & 495 & 539 \\
\hline CtpV & VGD & PVD & TGES & CPCAL & DKTGTLT & AAAVE & DTVK & MITGD & VAMVGDGVNDAPALVQADLGIAI \\
\hline Position & 282 & 295 & 313 & 416 & 460 & 490 & 589 & 611 & 655 \\
\hline KdpB & AGE & SVD & TGES & - & DKTGTIT & KGAAA & DEMR & MITGD & VAMTGDGTNDAPALAQADVGVAM \\
\hline Position & 153 & 169 & 176 & - & 324 & 417 & 485 & 496 & 540 \\
\hline HM Consensus & VGD & PAD & TGES & CPCAL & DKTGTLT & AAAVE & DTLR & LLTGD & VAMVGDGINDAPALAAADLGIAM \\
\hline H AM Consensus & PGD & PAD & TGES & PEGL & DKTGTLT & KGAPE & DTPR & LITGD & VAMTGDGVNDAAALRXADIGVAM \\
\hline \multicolumn{10}{|c|}{$\begin{array}{l}\text { Figure } \mathbf{5} \text { Conserved motifs for the P-type ATPases identified in } \mathbf{M} \text {. tuberculosis } \mathbf{H} 3 \mathbf{7 R v} \text {. The conserved motifs were compared with the } \\
\text { motifs characteristic of the P-type ATPase superfamily. Identical residues, conserved substitutions, semi-conserved substitutions and unrelated } \\
\text { residues are indicated in red, blue, green and black, respectively. }\end{array}$} \\
\hline
\end{tabular}




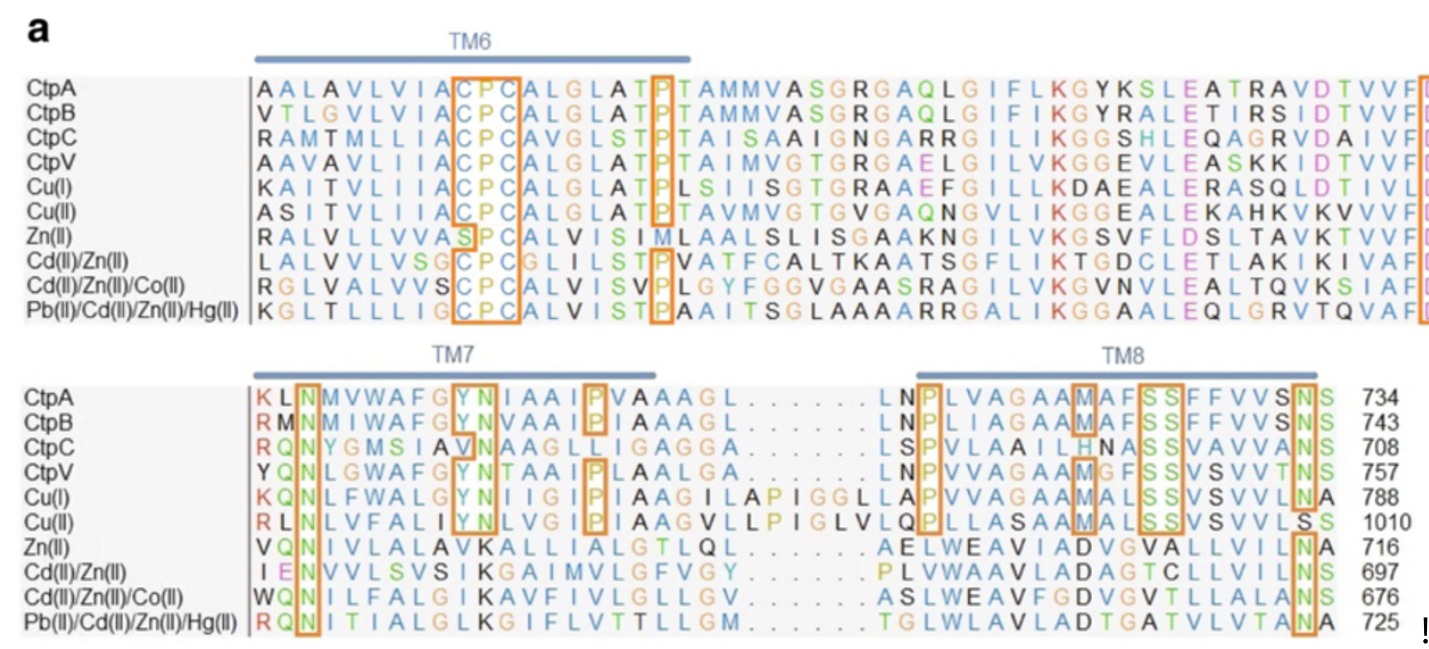

\section{b}

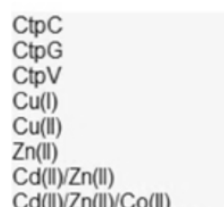

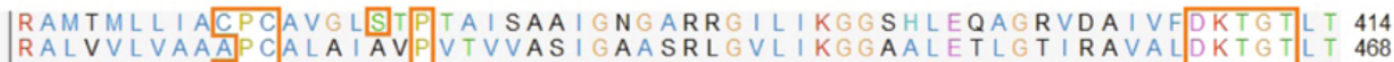
AAVA V L I I ACP C A LGLATP TA I MVG TGRGAELGILVKGGEVLEASKKIDTVVFDKTGTLT 466 KA | TVL I I ACPCALGLATPLS I ISGTGRAAEFG I LLKDAEALERASQLDT IVLDKTGTLT 492 AS I TVLI I ACPCALGLATPIAVMVGTGVGAQNGVLIKGGEALEKAHKVKVVVFDKTGTLT 704 RALVLLVVASPCALVISIMLAALSLISGAAKNGILVKGSVFLDSLTAVKTVVFDKTGTLT 431 LALVVLVSGCPCGLI L STIPVATFCALTKAATSGFL IKTGDCLETLAKIKIVAFDKTGTIT 403

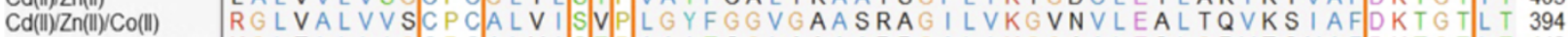

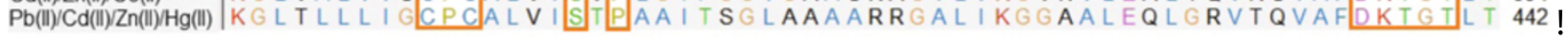

\begin{tabular}{|c|c|c|c|}
\hline & TM7 & TM8 & \\
\hline 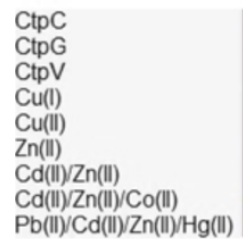 & 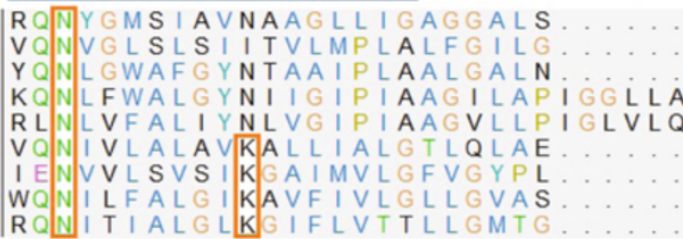 & 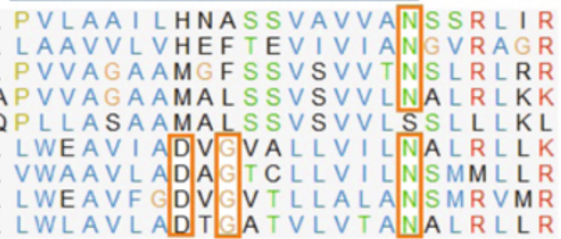 & $\begin{array}{l}713 \\
754 \\
762 \\
793 \\
1015 \\
721 \\
702 \\
681 \\
730\end{array}$ \\
\hline
\end{tabular}

C

CtpD

CtpJ

$\mathrm{Cu}(\mathrm{l})$

$\mathrm{Cu}(\mathrm{II})$

$\mathrm{Cd}($ II) $/ \mathrm{Zn}(\mathrm{II})$

$\mathrm{Cd}$ (II) $/ \mathrm{Zn}$ (II)/Co(II)

$\mathrm{Pb}(\mathrm{II}) / \mathrm{Cd}(\mathrm{II}) \mathrm{Zn}(\mathrm{II}) / \mathrm{Hg}$

TM6

IRAMTFM I VASPCA VVLATMPPLLSAIANAGRHGVLVKSAVVVERLADTSIVALDKTGTLT 353 RAMTFM VASPCAVVLATMPDI A I ANAGRHGVLAKSAIVMEQLGTTTRIAFDKTGTLT 346 KAI TVLI I ACPCALGLATPLSI ISGTGRAAEFGILLKDAEALERASQLDTIVLDKTGTLT 492 $\begin{array}{lll}K A & T V L \\ A S & T V L & A C P C A\end{array}$ RALVLLVVASPCALVISIMLAALSLISGAAKNGILVKGSVFLDSLTAVKTVVFDKTGTLT 431 LALVVLVSGCPCGLILSTPVATFCALTKAATSGFLIKTGDCLETLAKIKIVAFDKTGTIT 403 RGLVALVVSCPCALVISVPLGYFGGVGAASRAGILVKGVNVLEALTQVKS IAFDKTGTLT 394 KKGLTLLLIGCPCALVISTPAA I TSGLAAAARRGALIKGGAALEQLGRVTQVAFDKTGTLT 442 !

TM7

$\mathrm{CtpD}$

CtpJ

Cu(l)

$\mathrm{Cu}(\mathrm{ll})$

Zn(II)

$\mathrm{Cd}($ III) $/ \mathrm{Zn}(\mathrm{II})$

$\mathrm{Cd}$ (II)/Zn(II)/Co(II)

$\mathrm{Pb}(\mathrm{ll}) / \mathrm{Cd}(\mathrm{II}) \mathrm{Zn}(\mathrm{II}) / \mathrm{Hg}(\mathrm{II})$

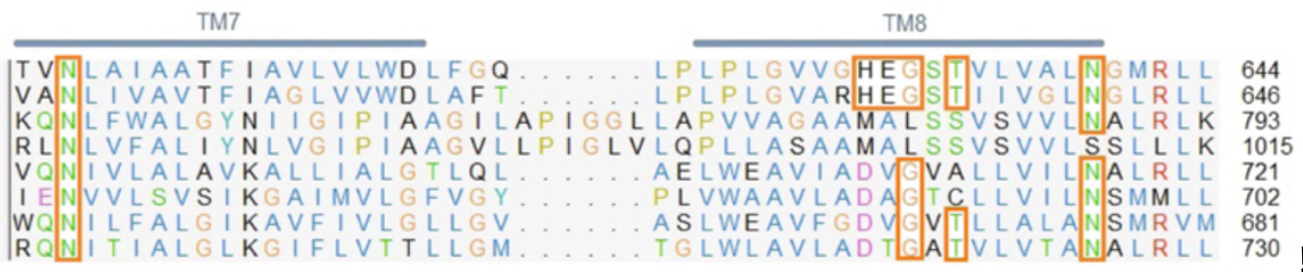

Figure 6 Alignments of consensus sequences from characterized $\mathrm{P}_{\mathrm{IB}}$ ATPases, with the HM P-type ATPases from M. tuberculosis H37Rv. (a) $\mathrm{P}_{\mathrm{IB}-1}$ motifs, (b) $\mathrm{P}_{\mathrm{IB}-2}$ motifs and (c) $\mathrm{P}_{\mathrm{IB}-4}$ motifs. 
Table 1 Additional conserved motifs observed in heavy metal transporter P-type ATPases

\begin{tabular}{|c|c|c|c|c|c|}
\hline $\begin{array}{l}\text { Phylogenetic } \\
\text { subgroup }\end{array}$ & $\begin{array}{c}\text { Cation } \\
\text { specificity }\end{array}$ & TMS6 & TMS7 & TMS8 & MTBC pump \\
\hline \multirow[t]{3}{*}{$\overline{\mathrm{P}_{\mathrm{IB}-1}}$} & $\mathrm{Cu}^{+}$ & CPC & YN & MXXSS & CtpA \\
\hline & & & & & CtpB \\
\hline & & & & & CtpV \\
\hline$\overline{P_{\mathrm{IB}-2}}$ & $\mathrm{Zn}^{2+}$ & $\mathrm{CPC}(\mathrm{X})_{4} \mathrm{~S}$ & K & $\mathrm{DG}$ & $\mathrm{CtpC}^{*}$ \\
\hline$\overline{\mathrm{P}_{\mathrm{IB}-3}}$ & $\mathrm{Cu}^{2+}$ & $\mathrm{CPH}$ & YN & MXXS & - \\
\hline \multirow[t]{2}{*}{$\overline{P_{\text {IB-4 }}}$} & $\mathrm{Co}^{2+}$ & SCP & $\mathrm{N}$ & HEGT & CtpD * \\
\hline & & & & & CtpJ * \\
\hline$\overline{P_{\mathrm{IB}-5}}$ & Undetermined & TPCP & $\mathrm{QX}_{4} G \mathrm{X}_{3} \mathrm{SX}_{3} \mathrm{M}$ & $\mathrm{PX}_{5} \mathrm{QEX}_{2} \mathrm{DX}_{5} \mathrm{~N}$ & - \\
\hline
\end{tabular}

The additional motifs are located on TMS6, TMS7 and TMS8. *, pumps without a complete set of motifs (see discussion).

that each MTBC species has a consistent aggrupation of the 12 P-type ATPases involved in ion transport. In this context, M. tuberculosis strains H37Ra and H37Rv share identical sequences for P-type ATPases, facilitating subsequent genetic studies using the attenuated strain H37Ra. The large number of HM P-type ATPases expressed by the MTBC strongly suggests that they could be essential for the bacteria to counteract the increased level of HM accumulated by macrophages after infection with tubercle bacilli. Thus, compensatory ion transport strategies could be used by mycobacteria to survive in host cells.

The different bioinformatics approaches used in this work to analyze the P-type ATPases identified in the MTBC are in agreement with the initial classification from the HMM search. The results obtained show that $M$. tuberculosis has the following three groups of P-type ATPases: HM transporters (CtpA, CtpB, CtpC, CtpD, CtpG, CtpJ and CtpV), AEM transporters (CtpE, CtpF, $\mathrm{CtpH}$, and $\mathrm{CtpI}$ ) and the $\mathrm{KdpB}$ protein, which corresponds to the $\beta$ subunit of a multimeric $\mathrm{K}^{+}$ATPase transporter exclusive to prokaryotes. Hydrophobicity analysis identified $\alpha$-helix type TMS grouped into the following three topological types: type I (HM group), type II (AEM group) and type III (KdpB group). Interestingly, we report a possible mis-annotation for $\mathrm{CtpH}$ and CtpI in the TCDB Database, where they are classified as FUPA 24 type with two TMS, unlike the ten TMS identified for these unusually large transporters in this work. Finally, a counterpart of non-catalytic $\beta$ subunits of $\mathrm{Na}^{+} / \mathrm{K}^{+}$or $\mathrm{H}^{+} / \mathrm{K}^{+}$ATPases does not exist within the MTBC proteomes.

\section{Competing interest}

There are no conflicts of interest to declare.

\section{Authors' contributions}

LN-A, AL-T, MP-R, JC-B, L-M S, DL, LM-R and CY-S wrote the manuscript, validated the tools and carried out the data analysis and interpretation. LN-A, $A L-T, M P-R$ and JC-B contributed to the methodological design, supervised its development and critically revised the manuscript's content. All authors read and approved the final version of the manuscript.

\section{Acknowledgements}

This work was supported by the Dirección de Investigación Bogotá (DIB)Universidad Nacional de Colombia, grants 12351, 12176, 11875, 11882 and Colciencias grant 13802. This research was supported in part by the Intramural Research Program of the National Institutes of Health, National Library of Medicine and National Center for Biotechnology Information.

Funding

This work was supported by the Dirección de Investigación Bogotá (DIB)Universidad Nacional de Colombia, grants 12351, 12176, 11875, 11882 and Colciencias grant 13802. This research was supported in part by the Intramural Research Program of the National Institutes of Health, National Library of Medicine and National Center for Biotechnology Information. The funding sources had no involvement in the study design; collection, analysis and interpretation of data; the writing of the manuscript or the decision to submit the article for publication.

\section{Author details}

${ }^{1}$ Chemistry Department, Faculty of Sciences, Universidad Nacional de Colombia, Bogotá, Colombia, Carrera 30 \# 45-03, Ciudad Universitaria, Bogotá, Colombia. ${ }^{2}$ Computational Biology Branch, NCBI, NLM, NIH, Bethesda, USA. ${ }^{3}$ PanAmerican Bioinformatics Institute, Santa Marta, Magdalena, Colombia.

Received: 25 June 2012 Accepted: 27 September 2012

Published: 3 October 2012

\section{References}

1. WHO: Global tuberculosis control: WHO report 2011. Switzerland: Publications of the World Health Organization; 2011:246.

2. McEvoy CR, et al: The role of IS6110 in the evolution of Mycobacterium tuberculosis. Tuberculosis (Edinb) 2007, 87(5):393-404.

3. Issa $R$, et al: Detection and discrimination of Mycobacterium tuberculosis complex. Diagn Microbiol Infect Dis 2012, 72(1):62-7.

4. Knechel NA: Tuberculosis: pathophysiology, clinical features, and diagnosis. Crit Care Nurse 2009, 29(2):34-43. quiz 44.

5. Tufariello JM, Chan J, Flynn JL: Latent tuberculosis: mechanisms of host and bacillus that contribute to persistent infection. Lancet Infect Dis 2003, 3(9):578-90.

6. Koul A, et al: The challenge of new drug discovery for tuberculosis. Nature 2011, 469(7331):483-90.

7. Nagata T, et al: Comparative molecular biological analysis of membrane transport genes in organisms. Plant Mol Biol 2008, 66(6):565-85.

8. Axelsen KB, Palmgren MG: Evolution of substrate specificities in the P-type ATPase superfamily. J Mol Evol 1998, 46(1):84-101.

9. Pedersen PL: Transport ATPases into the year 2008: a brief overview related to types, structures, functions and roles in health and disease. J Bioenerg Biomembr 2007, 39(5-6):349-55.

10. Rocafull MA, et al: Isolation and cloning of the $K_{+}-$independent, ouabaininsensitive Na+-ATPase. Biochim Biophys Acta 2011, 1808(6):1684-700. 
11. Pinoni SAL, A A: Na+ ATPase activities in chela muscle of the euryhaline crab Neohelice granulata: differential response to environmental salinity. J Exp Mar Bio Ecol 2009, 372(1-2):91-97.

12. Jorgensen PL: Purification and characterization of ( $\mathrm{Na}+, \mathrm{K}+)$-ATPase. V. Conformational changes in the enzyme Transitions between the $\mathrm{Na}$ form and the K-form studied with tryptic digestion as a tool. Biochim Biophys Acta 1975, 401(3):399-415.

13. Jorgensen $\mathrm{PL}$, Hakansson $\mathrm{KO}$, Karlish SJ: Structure and mechanism of $\mathrm{Na}$, K-ATPase: functional sites and their interactions. Annu Rev Physiol 2003, 65:817-49.

14. Xu C, et al: A structural model for the catalytic cycle of $\mathrm{Ca}(2+)$-ATPase. J Mol Biol 2002, 316(1):201-11.

15. Palmgren MG, Nissen P: P-type ATPases. Annu Rev Biophys 2011, 40:243-66.

16. Kuhlbrandt W: Biology, structure and mechanism of P-type ATPases. Nat Rev Mol Cell Biol 2004, 5(4):282-95.

17. Bublitz M, et al: In and out of the cation pumps: P-type ATPase structure revisited. Curr Opin Struct Biol 2010, 20(4):431-9.

18. Uniprot. cited 2011; Available from: http://www.uniprot.org/.

19. Pirovano W, Feenstra KA, Heringa J: PRALINETM: a strategy for improved multiple alignment of transmembrane proteins. Bioinformatics 2008, 24(4):492-7.

20. Eddy SR: Profile hidden Markov models. Bioinformatics 1998, 14(9):755-63.

21. Zhang Y: I-TASSER server for protein 3D structure prediction. BMC Bioinforma 2008, 9:40.

22. Thever MD, Saier MH Jr: Bioinformatic characterization of P-type ATPases encoded within the fully sequenced genomes of 26 eukaryotes. J Membr Biol 2009, 229(3):115-30.

23. Chan $\mathrm{H}$, et al: The P-type ATPase superfamily. J Mol Microbiol Biotechnol 2010, 19(1-2):5-104.

24. Cole ST: Deciphering the biology of Mycobacterium tuberculosis from the complete genome sequence. Nature 1998, 393:537.

25. Botella $\mathrm{H}$, et al: Mycobacterial $\mathrm{p}$ (1)-type ATPases mediate resistance to zinc poisoning in human macrophages. Cell Host Microbe 2011, 10(3):248-59.

26. Bramkamp M, Altendorf K, Greie JC: Common patterns and unique features of P-type ATPases: a comparative view on the KdpFABC complex from Escherichia coli (Review). Mol Membr Biol 2007, 24(56):375-86.

27. Lewinson O, Lee AT, Rees DC: A P-type ATPase importer that discriminates between essential and toxic transition metals. Proc Natl Acad Sci U S A 2009, 106(12):4677-82.

28. Argüello JM: Identification of lon-Selectivity Determinants in Heavy-Metal Transport P1B-type ATPases. J Membr Biol 2003, 195:93-108.

29. Argüello JM, Eren E, González-Guerrero M: The structure and function of heavy metal transport P1B ATPases. Biometals 2007, 20:233-248.

30. Argüello JM, Gonzalez-Guerrero M, Raimunda D: Bacterial transition metal P(1B)-ATPases: transport mechanism and roles in virulence. Biochemistry 2011, 50(46):9940-9.

31. Ward SK, et al: CtpV: a putative copper exporter required for full virulence of Mycobacterium tuberculosis. Mol Microbiol 2010, 77(5):1096-1110.

32. Lewinson O, Lee AT, Rees DC: A P-type ATPase importer that discriminates between essential and toxic transition metals. Proc Natl Acad Sci 2009, 106(12):4677-4682.

33. Futai M, Wada Y, Kaplan JH: Handbook of ATPases: Biochemistry, Cell Biology, Pathophysiology. 1 ed. Weinheim: WILEY-VCH Verlag GmbH \& Co. KGaA; 2004.

34. Schnappinger D, et al: Transcriptional Adaptation of Mycobacterium tuberculosis within Macrophages: Insights into the Phagosomal Environment. J Exp Med 2003, 198(5):693-704

35. Kumar $M$, et al: Identification of Mycobacterium tuberculosis genes preferentially expressed during human infection. Microb Pathog 2011, 50(1):31-8.

36. Waddell SJ, et al: The use of microarray analysis to determine the gene expression profiles of Mycobacterium tuberculosis in response to anti-bacterial compounds. Tuberculosis (Edinb) 2004, 84(3-4):263-74.

37. Bacon J, et al: The influence of reduced oxygen availability on pathogenicity and gene expression in Mycobacterium tuberculosis. Tuberculosis (Edinb) 2004, 84(3-4):205-17.

38. Sherman DR, et al: Regulation of the Mycobacterium tuberculosis hypoxic response gene encoding alpha -crystallin. Proc Natl Acad Sci U S A 2001, 98(13):7534-9.
39. Kendall SL, et al: The Mycobacterium tuberculosis dosRS two-component system is induced by multiple stresses. Tuberculosis (Edinb) 2004, 84(3-4):247-55.

40. Voskuil Ml, et al: Inhibition of respiration by nitric oxide induces a Mycobacterium tuberculosis dormancy program. J Exp Med 2003, 198(5):705-13.

doi:10.1186/1472-6807-12-25

Cite this article as: Novoa-Aponte et al:: In silico identification and characterization of the ion transport specificity for P-type ATPases in the Mycobacterium tuberculosis complex. BMC Structural Biology 2012

12:25.

\section{Submit your next manuscript to BioMed Central and take full advantage of:}

- Convenient online submission

- Thorough peer review

- No space constraints or color figure charges

- Immediate publication on acceptance

- Inclusion in PubMed, CAS, Scopus and Google Scholar

- Research which is freely available for redistribution

Submit your manuscript at www.biomedcentral.com/submit
C) Biomed Central 